\title{
IMPACTO AMBIENTAL GENERADO POR LAS \\ ACTIVIDADES QUÍMICAS DE PRODUCCIÓN Y USO DE CLOROFLUOROCARBONOS
}

Jaime Enrique Guerrero Santafé

Universidad Pedagógica Nacional.

\begin{abstract}
The purpose of the present publication is to discuss and analyzed the transcendence and magnitude of the impact produced by chemical activities over the environment, using a specific problem, the reduction of stratospheric ozone layer caused by the use of chlorofluorocarbons.
\end{abstract}

\begin{abstract}
RESUMEN
El objetivo de la presente publicación es discutir y analizar la trascendencia y magnitud del impacto producido por las actividades químicas en el ambiente utilizando un problema específico, la reducción del ozono estratosférico, ocasionada por el uso de clorofluorocarbonos.
\end{abstract}

\section{INTRODUCCIÓN}

Las actividades humanas concebidas como las diversas formas de interacción entre lo antrópico y lo natural, se refieren a las relaciones de los grupos humanos con los componentes de los ecosistemas terrestres, en la búsqueda de alternativas para satisfacer necesidades de subsistencia y perdurabilidad de la vida en el planeta.

Para interpretar la incidencia y significado de las actividades humanas y químicas en el ambiente y su relación con la problemática de la cotidianidad, se analizarán las alteraciones y el impacto que ha venido causando en el macrosistema terrestre, la producción y el uso de clorofluorocarbonos desde 1930. 


\section{OZONO ESTRATOSFÉRICO Y VIDA EN EL PLANETA}

El ozono es una forma alotrópica del oxigeno y constituye una de las tres formas en que pueden conformarse los átomos de oxígeno como $\mathrm{O}, \mathrm{O} 2$ y $\mathrm{O} 3$, siendo el Oxígeno molecular la forma más abundante en la atmósfera terrestre.

El ozono es un gas de color azul claro a temperatura ambiente y se descompone para formar oxígeno. Se encuentra fundamentalmente en la estratosfera terrestre por encima de 10.000 metros de la superficie y es esencial para garantizar la existencia de vida en el planeta, al absorber la peligrosa forma de radiación ultravioleta proveniente del sol, convirtiéndola en calor.

A menores alturas (troposfera), el ozono puede producirse por descargas eléctricas durante las tormentas y por actividades humanas como la iniciación de motores eléctricos, el funcionamiento estufas eléctricas, de ambientadores, equipos de calefacción y otros. Es el componente principal de "Smog fotoquímico" presente en las ciudades y sitios de gran circulación vehicular.

El ozono es la sustancia con mayor poder de oxidación después del flúor, propiedad que le confiere muchas aplicaciones pero también muchos problemas, especialmente a nivel troposférico.

En razón de su alta capacidad de oxidación, puede oxidar iones metálicos, alquenos, aminas, sulfuros, cianuros, fosfinas y otros grupos orgánicos. Puede oxidar y destruir los aminoácidos, los componentes claves de todas las proteínas los virus y las bacterias; aspecto que se aprovecha para el tratamiento y purificación del agua. Las cervecerías esterilizan el agua para elaborar sus diversas bebidas alcohólicas y refrescantes y dejan una cantidad residual de ozono en la solución para una posterior esterilización después de sellado el recipiente.

Muchas industrias utilizan ozono para tratar sus aguas residuales y remover los materiales tóxicos, antes de ser descargados. El uso más amplio del ozono está relacionado con la remoción de las especies químicas responsables del olor. Los grupos funcionales responsables de los olores desagradables en el agua, como los alquenos, sulfuros y aminas son degradados por el ozono.

El ozono posee olor fuerte, es irritante para las membranas y las mucosas humanas y animales y puede destruir las hojas de las plantas en razón de poder oxidar los aminoácidos contenidos en los tejidos vegetales. La inhalación en cantidad suficiente puede originar toxicidad. Los niveles de permisibilidad para ozono en sitos de trabajo es de $0.080 \mathrm{ppm}$ (mg/l) (Jacob, 1991). Existen dos clases de situaciones ambientales en relación el ozono, originadas por la producción y uso de clorofluorocarbonos: una relacionada con el ozono troposférico, desde superficie de la tierra hasta 15 Kilómetros de altura, y otra con el ozono estrastoférico, desde 10 Kilómetros hasta 55 kilómetros de altura. En la troposfera terrestre se encuentra el aire con sus componentes $\mathrm{N}_{2}, \mathrm{O}_{2}, \mathrm{CO}_{2}$, las nubes y otras especies en menor concentración y los demás factores del clima y aquí los vientos, las tormentas y demás sistemas climáticos se mezclan con los gases atmosféricos en forma horizontal y vertical. 
En la estratosfera los vientos en pequeñas capas se mueven alrededor de la tierra con poca mezcla vertical. La naturaleza de la capa estratosferica es el resultado de la conversión de la radiación ultravioleta en calor por las reacciones del ozono, por esta razón a 50 Kilómetros de altura de la superficie de la tierra (estratosfera), la temperatura es de $-2^{\circ} \mathrm{C}$ en contraste con la temperatura de $-56^{\circ} \mathrm{C}$ a 15 Kilómetros de altura (troposfera), (Jacob, 1991) por la poca mezcla vertical en esta zona, el aire se vuelve menos denso y tiende a elevarse. En la troposfera es lo contrario y por lo tanto la mayor parte de la radiación solar es absorbida por la superficie de la tierra.

En consideración a que la troposfera terrestre se inicia en la superficie de la tierra, la cual soporta todos los organismos, el ozono en esta región de la atmósfera es perjudicial para los seres vivientes.

\section{OZONO TROPOSFÉRICO}

Como se mencionó la mayoría del ozono troposférico se obtiene por la reacción de los contaminantes del aire producidos principalmente por la combustión interna de motores de vehículos y aparatos eléctricos. Los contaminantes se forman cuando el oxígeno del aire y el nitrógeno son calentados y reaccionan para formar mezclas de gases de óxidos de nitrógeno $\left(\mathrm{NO}_{\text {y NO}}\right.$ ) Los cuales también son los responsables del smog:

$$
\mathrm{N}_{2}(\mathrm{~g})+\mathrm{O}_{2}(\mathrm{~g}) \rightarrow 2 \mathrm{NO}(\mathrm{g}) \text { y } 2 \mathrm{NO}(\mathrm{g})+\mathrm{O}_{2}(\mathrm{~g}) \rightarrow 2 \mathrm{NO}_{2}(\mathrm{~g})
$$

Estos indeseables productos de la combustión pueden seguir reaccionando en la tropósfera:

$$
\mathrm{NO}_{2}(\mathrm{~g}) \text { radiación visible } \mathrm{NO}(\mathrm{g})+\mathrm{O}(\mathrm{g})
$$

El oxígeno atómico (O.) producido puede reaccionar con el oxígeno molecular del aire y formar ozono: $\mathrm{O}(\mathrm{g})+\mathrm{O}_{2}(\mathrm{~g}) \rightarrow \mathrm{O}_{3}(\mathrm{~g})$. Para tener una idea de la magnitud de éstos procesos, recordemos que la masa molecular de cada una de las especies anteriores contiene el número de Avogadro de moléculas. Si se establecen loa mecanismos que puedan permitir la medición de las cantidades en kilogramos o en gramos de los gases indeseables producidos por Los miles de millones de motores y máquinas que operan en la tierra para diversas actividades, podremos tener una percepción más clara tanto cuantitativa como cualitativa del ozono producido en la tropósfera terrestre, de la magnitud del smog formado y de los efectos en la salud ambiental.

\section{OZONO ESTRATOSFÉRICO}

El ozono estrafosférico desempeña el poderoso papel de proteger la vida de los organismo del planeta. La capa de ozono en la estratosfera terrestre es como el paraguas protector de la tierra que retiene la peligrosa radiación ultravioleta que amenaza la vida de todas las especies.

El proceso de formación y de consumo del ozono estratosférico puede percibirse cuando el oxígeno molecular $\left(\mathrm{O}_{2}\right)$ absorbe radiaciones ultravioleta de alta energía y de longitud de onda por debajo de $242 \mathrm{~nm}\left(1 \mathrm{~nm}=10^{-9} \mathrm{M} 10^{-7} \mathrm{~cm}\right)$. 
$\mathrm{O}_{2}$ radiación ultravioleta $\rightarrow 20$ (g) (proceso de fotodisociación)
$\mathrm{n}<242 \mathrm{~nm}$
Se necesita energía

El oxígeno atómico $(\mathrm{O})$ reacciona con el oxígeno molecular $\left(\mathrm{O}_{2}\right)$ para formar ozono (O3): $\mathrm{O}_{2}(\mathrm{~g})+\mathrm{O} .(\mathrm{g}) \rightarrow \mathrm{O}_{3}(\mathrm{~g})$, se ibera energía.

El ozono formado reacciona con radiación ultravioleta de longitud de onda entre 200 y $300 \mathrm{~nm}$ y se fotodisocia en $\mathrm{O}_{2}$ y $\mathrm{O}$ y se ibera gran cantidad de calor.

$\mathrm{O}_{3}(\mathrm{~g}) \frac{\text { radiación ultravioleta }}{\mathrm{n}=200-300 \mathrm{~nm}} \rightarrow \mathrm{O}_{2}(\mathrm{~g})+\mathrm{O}(\mathrm{g})$ se libera calor

Las reacciones de formación y fotodisociación del ozono se pueden asociar para poder analizar el papel crucial de protección del ozono en el ecosistema por la absorción de la peligrosa radiación ultravioleta y su conversión a calor (Jacob 1991).

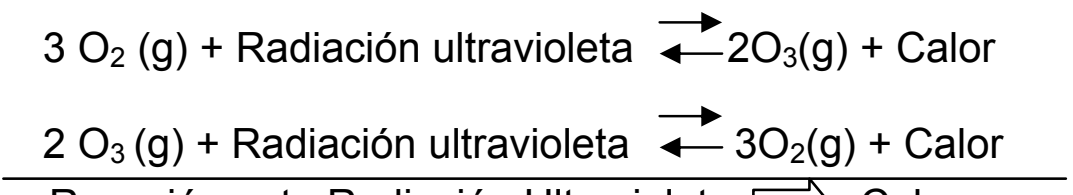

Reacción neta Radiación Ultravioleta $\Rightarrow$ Calor

La conversión de la radiación ultravioleta en calor por éste proceso es la más grande en la estratosfera superior, donde la intensidad de la radiación es también mayor. Disminuye a menores altitudes estrastosféricas a medida que la intensidad de la radiación disminuye y la concentración del ozono disminuye.

El gran riesgo ambiental de la radiación ultravioleta sobre la vida humana, de animales o de plantas se refiere a que la gran intensidad y poder de la radiación ultravioleta puede oscurecer, secar y envejecer la piel, puede causar úlceras y originar cáncer en los tejidos. La radiación también puede dañar y alterar las unidades claves del ácido desoxirribunocleico DNA, que contiene la información genética indispensable para la reproducción perpetuidad de vida de las especies. La exposición excesiva a radiación ultravioleta puede causar mutaciones, cáncer de piel y dañar los ojos. Por lo anterior se recomienda a los bañistas no exponerse a largos períodos de exposición solar.

\section{REDUCCIÓN DEL OZONO ESTRASTOSFERICO POR AERONAVES SUPERSÓNICAS Y POR EL USO DE CLOROFLUOROCARBONOS}

La Hipótesis que las actividades humanas pueden reducir la capa de ozono estrastosférico fue formulada a comienzos de la década del setenta. El proyecto de diseño y construcción de aviones comerciales supereconómicos que pudiesen volar en la estratosfera terrestre iniciada por los Norteamericanos (1971) y continuada y mantenida por Francia e Inglaterra condujo a la construcción del Concorde, aeronave que todavía realiza vuelos comerciales entre el continente Europeo y Norteamericano (Henriques 1972). Para este tipo de aeronaves es más económico volar en la estratosfera donde la densidad del aire es menor y recordando que existe muy poca mezcla vertical en ésta parte de la atmósfera, los gases emitidos por los exhostos de los aviones permanecen por más tiempo allí. (Chemical Engineering News 1971). 
Estos gases son principalmente óxidos de nitrógeno, los cuales pueden propiciar la destrucción del ozono.

$$
\begin{aligned}
& \mathrm{NO}(\mathrm{g})+\mathrm{O} 3(\mathrm{~g}) \rightarrow \mathrm{NO}_{2}(\mathrm{~g})+\mathrm{O} 2(\mathrm{~g})(1) \\
& \mathrm{NO}(\mathrm{g})+\mathrm{O}(\mathrm{g}) \rightarrow \mathrm{NO}(\mathrm{g})+\mathrm{O} 2(\mathrm{~g})(2) \\
& \mathrm{NO}(\mathrm{g})+\mathrm{O} 3(\mathrm{~g}) \rightarrow \mathrm{NO}_{2}(\mathrm{~g})+\mathrm{O} 2(\mathrm{~g})(1)
\end{aligned}
$$

La regeneración del NO en la segunda reacción puede repetirse muchas veces puesto que éste oxido de nitrógeno actúa como un catalizador y no se consume, razón por la cual propicia una reacción en cadena destruyendo el ozono y causando graves perjuicios al ecosistema terrestre. Una disminución del $1 \%$ de la capa de ozono resultaría en un $2 \%$ de cáncer de piel. (Jacob 1991). No podemos olvidar que los aviones concordes no son los únicos que vuelan en la estratosfera terrestre, también lo hacen las aeronaves de guerra de los países del mundo, quienes aprovechando la menor densidad atmosférica y la menor mezcla de gases encuentran en ésta región atmosférica el espacio propicio para que los aviones vuelen a dos o tres veces la velocidad del sonido y contribuyan a incrementar el riesgo ambiental.

En 1974, Mario Molina y Rowland Sherwood, presentaron una teoría en la cual describían cómo los ampliamente utilizados clorofluorocarbonos podían afectar y alterar las condiciones de vida del ambiente. (Molina, Rowland 1974). Algunos científicos de las industrias consideraron que ésta hipótesis no tenía sentido y el presidente de una industria de aerosoles le atribuyó razones políticas.

Los clorofluorocarbonos comenzaron a producirse por varias industrias norteamericanas desde 1930 y se creían como los compuestos ideales: contienen carbono y halógeno, no son inflamables, se usan para extinguidores, son muy estables y por esto se creyó que no reaccionaban ni producían otros productos indeseables; no son tóxicos y eran considerados ambientalmente seguros en la troposfera.

Molina y Rowland, propusieron que una vez que los clorofluorocarbonos hayan alcanzado la estratosfera estarían expuestos a la poderosa radiación ultavioleta ( $\mathrm{k}=175$ $220 \mathrm{~nm}$ ), cuya energía podría calcularse. En presencia de la radiación ultravioleta los clorofluorocarbonos son reactivos y causan daño a la capa de ozono. El mecanismo para la reacción que altera la capa de ozono involucra la exposición de las sustancias a la radiación, la disociación para formar dos radicales, el cloro atómico y el resto del compuesto padre y la reacción en cadena que destruye el ozono.

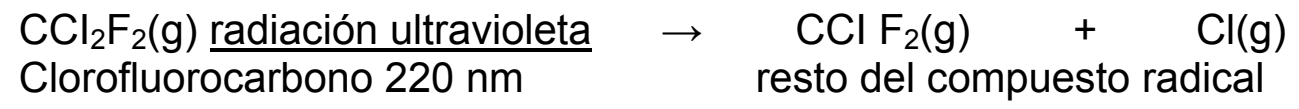

El radical $\mathrm{Cl}(\mathrm{g})$ formado puede actuar como catalizador en la destrucción del ozono presente en la estratosfera terrestre:

$$
\begin{aligned}
& \mathrm{Cl} .(\mathrm{g})+\mathrm{O}_{3}(\mathrm{~g}) \rightarrow \text { ClO. }(\mathrm{g})+\mathrm{O}_{2}(\mathrm{~g}) \\
& \text { Radical ozono Radical }
\end{aligned}
$$

ClO. $(\mathrm{g})+\mathrm{O}(\mathrm{g}) \rightarrow \mathrm{Cl} .(\mathrm{g})+\mathrm{O}_{2}(9)$

Radical ozono Radical

$$
\mathrm{Cl} .(\mathrm{g})+\mathrm{O}_{3}(\mathrm{~g}) \rightarrow \mathrm{ClO}(\mathrm{g})+\mathrm{O} 3(\mathrm{~g})
$$


La repetición de la reacción (3) indica la regeneración del radical $\mathrm{Cl}(\mathrm{g})$, y según los cálculos propuestos por los autores, podría repetirse la reacción (3) 100,000 veces con lo cual se destruirían 100.000 moléculas de ozono antes que sea consumido el radical por otras reacciones laterales, la reacción neta es:

$$
\begin{aligned}
& \mathrm{Cl}(\mathrm{g})+\mathrm{O}_{3}(9) \mathrm{ClO}(\mathrm{g})+\mathrm{O}_{2}(\mathrm{~g}) \\
& \mathrm{Cl}(\mathrm{g})+\mathrm{O}_{3}(\mathrm{~g}) \mathrm{ClO}(\mathrm{g})+\mathrm{O}_{2} \\
& \mathrm{O}_{3}(9)+\mathrm{O}(\mathrm{g}) \longrightarrow 2 \mathrm{O}_{2}(\mathrm{~g})
\end{aligned}
$$

\section{EL HUECO EN LA CAPA DE OZONO ESTRASTOSFERICO EN LA ANTARTIDA}

En 1985, Gardiner y Shanklin miembros del equipo Británico de Investigación en la Antártida reportaron en la revista Nature que la cantidad de ozono en la estratosfera sobre la Antártida había disminuido cerca del $50 \%$ al final del invierno y que a nivel global ésta pérdida representaba una disminución del 3-5\% y algunos estimaron en $5 \%$. La noticia causó pánico y gran preocupación y se habló por primera vez de un "Hueco en la capa de ozono estrastosférico".

Las especulaciones acerca del problema no se hicieron esperar por la aparición del hueco en la capa de ozono en la Antártida. Si no existen actividades humanas significativas en ésta región, qué ocasionaba las fluctuaciones en la capa de ozono en la Antártida? Cómo efectuaría las condiciones de vida, la reducción y el hueco en capa de ozono?

La primera y definitiva evidencia experimental que confirmó la teoría de Molina Rowland acerca de la destrucción del ozono estrastosférico ocasionada por las reacciones generadas por la fotodisociación de clorofluorocarbonos mediante la radiación ultravioleta, fueron el informe y publicación del equipo Británico de Investigación en la Antártida (Forman etal 1985).

Cómo explicar la gran pérdida de ozono al finalizar la estación de invierno y la recuperación parcial durante la primavera y el verano?

El continente Artico y antártico son muy diferentes a los demás del planeta. En el invierno y debido a la posición inclinada del eje terrestre con respecto al sol, se produce un periodo muy largo de oscuridad junio-agosto. En el verano es lo contrario. Al final del invierno cuando el sol regresa se forman los agujeros en la capa de ozono.

Los vientos antárticos se desplazan alrededor del polo sur. Estos vientos con circulación rotatoria constituyen lo que se conoce como vórtice circumpolar y transportan diferentes gases y mezclas, los cuales al encontrar temperaturas de $-85^{\circ} \mathrm{C}$, encuentran condiciones adecuadas para concentrarse y ubicarse sobre ésta región y algunos a éstas bajas temperaturas pueden formar sólidos hidratados, los cuales se fotodisocian al regresar la luz solar produciendo los radicales responsables del ataque al ozono y de la reacción en cadena.

Sí la reacción entre el $\mathrm{ClO}(\mathrm{g})$ con el $\mathrm{O}(\mathrm{g})$, produce $\mathrm{Cl}(\mathrm{g})$, el responsable del ataque al ozono pudiese evitarse, la acción catalítica del radical Cl podría detenerse. Cómo? Si el $\mathrm{NO}_{2}$ estuviese presente en la estratosfera. El $\mathrm{NO}_{2}$ producido por la combustión en motores 
o por combustión de plantas por biodegradación, puede reaccionar con el radical 010 para formar el nitrato de cloro $\mathrm{ClONO}_{2}(\mathrm{~g})$ que es menos riesgoso yen vez de destruir 100,000 moléculas de ozono, se destruiría solo una.

$\mathrm{Cl} .(\mathrm{g})+\mathrm{NO}_{2}(\mathrm{~g}) \rightarrow \mathrm{Cl} . \mathrm{NO}_{2}(\mathrm{~g})$ el cual se constituye en trampa para el $\mathrm{Cl} .(\mathrm{g})$ pues detiene su efecto catalítico. Otro mecanismo sería la reacción entre el cloro con el agua:

$$
\mathrm{Cl} .(\mathrm{g})+\mathrm{H}_{2} \mathrm{O}(\mathrm{g}) \rightarrow \mathrm{HCl}(\mathrm{g})+\mathrm{O} \cdot \mathrm{H}(\mathrm{g})
$$

Se cree que estas reacciones para detener el efecto catalítico del cloro se dan en la mayor parte de la estratosfera y por esta razón no se ha observado destrucción significativa del ozono en el resto del macrosistema terrestre. Las drásticas condiciones climáticas de la Antártida, la hacen vulnerable y contribuyen a que estos procesos tengan lugar.

$$
\text { La reacción entre el } \mathrm{ClONO}_{2}(\mathrm{~g})+\mathrm{HCl}(\mathrm{g}) \underset{\text { Invierno }}{-\frac{85^{\circ} \mathrm{C}}{\text { Inverno }}} \rightarrow \mathrm{Cl}_{2}(\mathrm{~g})+\mathrm{HNO}_{3} \cdot 3 \mathrm{H}_{2} \mathrm{O}(\mathrm{g})
$$

Puede dar origen a formas catalíticas las cuales pueden destruir las trampas que controlan éste comportamiento del $\mathrm{Cl}(\mathrm{g})$

$$
\begin{aligned}
& \mathrm{ClONO}_{2}(\mathrm{~g})+\mathrm{HCl}(\mathrm{g}) \underline{\text { invierno }} \rightarrow \mathrm{Cl}_{2}(\mathrm{~g})+\mathrm{HNO}_{3}(\mathrm{~s}) \\
& \mathrm{ClONO}_{2}(\mathrm{~g})+\mathrm{H}_{2} \mathrm{O}(\mathrm{g}) \underline{\text { invierno }} \rightarrow \mathrm{HOCl}(\mathrm{g})+\mathrm{HNO}_{3}(\mathrm{~s})
\end{aligned}
$$

El regreso de la luz solar después del oscuro invierno antártico, hace que $\mathrm{HOCl}(\mathrm{g})$ y el $\mathrm{Cl}_{2}(9)$ pueden fotodisociarse formando los radicales $\mathrm{Cl}(\mathrm{g})$ que destruyen el ozono:

$$
\begin{aligned}
& \mathrm{Cl}_{2}(9) \underline{\text { radiación }} \rightarrow 2 \mathrm{Cl}(\mathrm{g}) \\
& \mathrm{HOCl}(\mathrm{g}) \text { radiación } \rightarrow \mathrm{OH}(\mathrm{g})+\mathrm{Cl}^{\circ}(\mathrm{g})
\end{aligned}
$$

\section{ESTRATEGIAS Y ACCIONES FRENTE AL PROBLEMA}

Aunque la teoría Molina_Rowland fue propuesta en 1974, por intereses económicos, solo se tomaron acciones en 1979. En 1974, el consejo prodefensa de los recursos Naturales solicitó a la comisión de Seguridad de los Productos al Consumidor abolir el uso del clorofluorocarbonos en los aerosoles, esta comisión le solicitó a la Agencia de Protección ambiental (EPA), la cual respondió: "cuidamos la parte baja de la (tropósfera) atmósfera y éste es un problema de la atmósfera superior (estratosfera). Otras instituciones como la Administración Atmosférica y Oceánica Nacional, comentó que el asunto era muy especulativo. Solo la presión continuada de los grupos ambientales pudo conseguir la prohibición de los clorofluorocarbonos en los aerosoles en 1979. La concentración del ozono cerca de la región polar de acuerdo a los estudios realizados por los grupos de investigadores vía satélite se estimó ser cerca de la mitad de la concentración de ozono en la década anterior, 300 unidades Dobson (3.00 mm 1985), comparado con 600 unidades Dobson (1975), se refiere al espesor de la capa de ozono que resultaría si todo el ozono en la atmósfera se recolectara en una capa delgada a 1 atmósfera de presión y 000. La fotografía del satélite reveló que existen diferentes espesores de la capa de ozono sobre la Antártida, que van desde $4.75 \mathrm{~mm}$ a solo 1.00 
$\mathrm{mm}$, de espesor. (NASA total Mapping Spectrometer (toms) Instrument on Nasas Ninibas - 7 Satellite 1985).

Pero el hecho más contundente para tomar decisiones definitivas ocurrió con los hallazgos del Equipo de Investigación Británico en la Antártida en 1985. Los resultados dramáticos acerca de la pérdida del $50 \%$ del ozono Antártico, después del invierno, llevaron a las Naciones Unidas a construir una política la cual se consolidó bajo el nombre de Protocolo de Montreal para controlar las sustancias que reducen la capa de ozono.

La prohibición de producción de artículos de espumas de plástico por las industrias para buscar productos alternativos, a los talleres de servicio para reciclar los clorofluorocarbonos del aire acondicionado, los aerosoles, desodorantes y colonias que puedan contener o ayudar a los clorofluorocarbonos, debe abolirse o buscar sustitutos seguros. Las firmas Toyota, Nissan y Honda prometieron que antes de terminar este siglo XX, los sistemas de aire acondicionado no contendrán clorofluorocarbonos (Miller 1992).

La vida media de la mayoría de los clorofluorocarbonos es demasiado larga y por lo tanto este es un factor que contribuye a incrementar el riesgo ambiental para la vida en el planeta.

En 1987, se reunieron 28 países en Montreal, elaboraron y afirmaron un protocolo para reducir y abolir ocho de los clorofluorocarbonos más riesgosos. En 1990, cuatro países habían firmado y más tarde se unieron otros países. Si se cumple el Protocolo de Montreal, se reducirán las emisiones de Clorofluorocarbonos en un 35\% entre 1989 y el año 2000. Se evitarán 137 millones de cánceres de piel, 27 millones de muertes por dichos cánceres y 1.2 millones de cataratas (Miller 1992).

La búsqueda de productos alternos seguros para todos los materiales que reducen y dañan la capa de ozono es difícil pero no imposible y los científicos han buscado reemplazar los clorofluorocarbonos (CIFC) y los HidroFluorocarbonos (HFC) por Fluorocarbonos (CFC), teniendo en cuenta que reaccionen en la troposfera y no en la estratosfera.

- Halon: compuestos que contienen Bromo y que son usados como extinguidores

La Química atmosférica de los hidrocarbonos y de los hidroclorofluorocarbonos es de gran importancia para todos aunque estos compuestos no amenazan la capa de ozono. La fotooxidación de éstos compuestos y los efectos de la fotooxidación de sus productos son de gran relevancia; por ejemplo el compuesto (sustituto) $\mathrm{CF}_{3} \mathrm{CH}_{2} \mathrm{~F}$ se ha estudiado para analizar sus reacciones de eliminación en el ámbito de la troposfera y ser si afecta el ozono troposférico o no.

El compuesto reacciona cpon el radical hidroxil $(\mathrm{OH})$ :

$\mathrm{CF}_{3} \mathrm{CH}_{2} \mathrm{~F}+\mathrm{HO} . \rightarrow \mathrm{OH}_{3} \mathrm{CHF} .+\mathrm{H}_{2} \mathrm{O}$

El radical producido forma un radical peroxi con el oxígeno molecular (O2):

$\mathrm{CF}_{3} \mathrm{CHF} .+\mathrm{O}_{2}+\mathrm{H} \rightarrow \mathrm{CF}_{3} \mathrm{CHFO}_{2}{ }^{\circ} .+\mathrm{H}$

y el radical reacciona con el NO: 
$\mathrm{CF}_{3} \mathrm{CHFO}_{2}{ }^{\circ} .+\mathrm{NO} \rightarrow \mathrm{CF}_{3} \mathrm{CHFO} .+\mathrm{NO}_{2}$

El producto puede descomponerse o reaccionar con el $\mathrm{O}_{2}$

$\mathrm{CF}_{3} \mathrm{CHFO} \rightarrow \mathrm{CF}_{3}+\mathrm{HC}(\mathrm{O}) \mathrm{F}$

$\mathrm{CF}_{3} \mathrm{CHFO}+\mathrm{O}_{2} \rightarrow \mathrm{OF}_{3} \mathrm{O}(\mathrm{O}) \mathrm{F}+\mathrm{HO}_{2}{ }^{0}$

\begin{tabular}{|c|c|c|c|c|c|}
\hline FORMULA & SIMBOLO & $\begin{array}{l}\text { VIDA AÑOS } \\
\text { MEDIA } \\
\text { ATMOSFERA }\end{array}$ & usos & $\begin{array}{l}\text { IMPACTO } \\
\text { AMBIENTAL }\end{array}$ & SUSTITUTOS \\
\hline $\mathrm{CCl} 3 \mathrm{~F}$ & CFC -11 & 75 & $\begin{array}{l}\text { Espumas, aire } \\
\text { acondicionado, } \\
\text { refrigeración }\end{array}$ & $\begin{array}{l}\text { Destrucción ozono, } \\
\text { grave riesgo ambiental }\end{array}$ & $\mathrm{CHCl}_{2} \mathrm{CF}$ \\
\hline $\mathrm{CCL}_{2} \mathrm{~F}_{2}$ & CFC -12 & 111 & $\begin{array}{l}\text { Espumas, } \\
\text { refracción, aire } \\
\text { acondicionado, } \\
\text { aerosoles }\end{array}$ & $\begin{array}{l}\text { Destrucción ozono, } \\
\text { grave riesgo ambiental }\end{array}$ & $\mathrm{CH}_{2} \mathrm{FCF}_{3}$ \\
\hline $\begin{array}{l}\mathrm{CCl}_{2} \mathrm{FCClF}_{2} \\
\mathrm{CClF}_{2} \\
\mathrm{CClF}_{2}\end{array}$ & $\begin{array}{l}\text { CFC - } 113 \\
\text { CfC }-114\end{array}$ & $\begin{array}{l}90 \\
185\end{array}$ & $\begin{array}{l}\text { Espumas, } \\
\text { Disolventes, } \\
\text { refrigeración, aire } \\
\text { acondicionado }\end{array}$ & $\begin{array}{l}\text { Destrucción ozono, } \\
\text { grave riesgo ambiental }\end{array}$ & $\begin{array}{l}\mathrm{CF}_{3} \mathrm{CF}_{2} \mathrm{CHCl}_{2} \\
\mathrm{CHClFCF}_{3}\end{array}$ \\
\hline $\mathrm{CBrClF}_{2}$ & Halón - 1211 & 25 & Extinguidores & $\begin{array}{l}\text { Destrucción ozono, } \\
\text { grave riesgo ambiental }\end{array}$ & $\mathrm{CHCl}_{2} \mathrm{~F}_{3}$ \\
\hline $\begin{array}{l}\mathrm{CBrF}_{3} \\
\mathrm{CClF}_{2} \mathrm{CF}_{3}\end{array}$ & $\begin{array}{l}\text { Halón - } 1301 \\
\text { CFC - } 115\end{array}$ & $\begin{array}{l}110 \\
380\end{array}$ & $\begin{array}{l}\text { Extinguidores, } \\
\text { refrigeración, aire } \\
\text { acondicionado }\end{array}$ & Riesgo ambiental & $\begin{array}{l}\mathrm{CF}_{3} \mathrm{CHF}_{2} \\
\mathrm{CF}_{2} \mathrm{CF}_{3}\end{array}$ \\
\hline $\mathrm{C}_{2} \mathrm{Br}_{2} \mathrm{~F}_{4}$ & Halón* - 2402 & Sin reportar & Extinguidores & Riesgo ambiental & En proceso \\
\hline
\end{tabular}

Algunas reacciones estrastosféricas se cree involucran radicales que contienen el grupo $\mathrm{CF}_{3}$ producido parlas hidrocluorocarbonos y hidroclorofluorocarbonos, pero se ha concluido que no representan amenaza para el ozono estratosférico (Manahan 1994).

El 11 de octubre de 1995, la Real Academia de Ciencias de Suecia otorgó el premio Nobel de Química a los doctores Paul Crutzen del Instituto Maxplank, por haber demostrado en 1970 cómo los óxidos de nitrógeno aceleraban la velocidad de reducción del ozono; a Mario Molina del M:I:T: por haber concluido que la industria química estaba dañando la capa de ozono y a Sherwood Rowland de University of California at Irvine quien con el Dr. Molina fueron duramente criticados por la industria química por los hallazgos sobre la capa de ozono.

La Academia de Ciencias de Suecia al otorgarles el premio Nobel dijo: "Por haber contribuido a Salvarnos de un problema ambiental global que pudo tener consecuencias catastróficas y porque han hecho posible la toma de decisiones para prohibir la liberación de gases que destruyen el ozono". (The New York Times National, 1995).

Las Evidencias presentadas por diferentes investigadores del mundo han comprobado que las actividades humanas si están alterando y modificando el ambiente y en algunos casos con consecuencias desastrosas e irreversibles con relación a la vida en el planeta. 
El conocimiento y comportamiento individual, grupal, institucional, nacional e internacional es clave para determinarlos impactos negativos y positivos de nuestras actividades como también las estrategias y acciones de manejo técnico y educativo que debe asumir la comunidad internacional para garantizar la sostenibilidad del macrosistema terrestre en pro de mantener la vida que tantos millones de años le tomó al ecosistema trabajar y sustentar.

\section{REFERENCIAS}

1. CHEMICAL, and Engineering News - SST: Treat to ozone: 1971.

2. FARMAN J. Gardiner B. Shanklin J. Large Losses of total ozone in Antártica Reveal Seasonal ClOx/Nox Interaction. Nature 1985.

3. HENRIQUES F.C. Environmental Aspects of the supersonic Transport, A Recomended Program for Research and Measurement - U.S. Department Commerce 1972.

4. JACOB T.A. Chlorofluorocarbons and the Hale in the ozone Layer. I.C.E. University of Wisconsin 1991.

5 MANAHAN S. Environmental Chemistry. Lewis Publishers. Sixth Edition 1994. P. 371

6. MILLER T. Ecología y Medio Ambiente. Editorial Iberoamérica. Séptima Edición 1992, p. 329.

7. MOLINA M.J. Rowland F.S. Stratospheric Sink for Chlorofluoromethanes: Chlorine Atom - Catalized Destruction of ozone. Nature 1974.

8. NEW YORK TIMES, Three Win Nobel Prize For Research on ozone. Octubre 12. 1995 A 12. 